\title{
Specialist registrar training: ideas for a five year programme
}

\author{
Lynn Williams, Paul Pritty, Demas Esberger
}

\begin{abstract}
Proposals for specialist registrar training in accident and emergency medicine in Mid-Trent Region are outlined. These may provide a framework for other schemes as well as stimulating further ideas.

(F Accid Emerg Med 1997;14:324-325)
\end{abstract}

Keywords: specialist registrar; training programme

The commissioning date for the specialist registrar grade in accident and emergency medicine was 1 July $1996 .^{1}$ Much discussion was necessary between postgraduate deans, consultants in the specialty, and existing trainees to agree the most effective way to implement the new programme.

If specialist registrar training is to be effective it must be well organised, flexible, and capable of evolution. We describe an outline of the proposals for training in Mid-Trent which may provide a framework for other schemes as well as stimulating further ideas. The MidTrent programme includes two hospitals, Derbyshire Royal Infirmary and Queen's Medical Centre, and currently has seven specialist registrars in post.

\section{Training objectives}

In order that specialist registrars have an informed understanding of what to expect of their training in accident and emergency medicine, the objectives of the programme have been clearly defined:

(1) to provide a comprehensive theoretical and practical training in all aspects of the specialty; (2) to develop in the specialist registrar the professional maturity required of a consultant in the specialty;

Accident and Emergency Department, Queen's Medical Centre, Nottingham Lynn Williams Demas Esberger

\section{Accident and} Emergency Department Derbyshire Royal Infirmary, Derby Paul Pritty

Correspondence to: Miss L Williams, Consultant, Accident and Emergency Department, Queen's Medical Centre, Nottingham NG7 2UH

Accepted for publication 2 May 1997

(3) to identify and strengthen a specialist registrar's areas of interest and expertise;

(4) to prepare the specialist registrar for entry to the Fellowship examination in accident and emergency medicine.

\section{Supervision of the training programme}

One consultant has been appointed as programme director to oversee all aspects of training in both departments. Each department has an educational supervisor responsible for coordinating local training activities. Each trainee is assigned a named consultant as a trainer.

\section{Appraisal of trainees}

The overall objective of the appraisal mechanism is to assist specialist registrars in achieving their full potential. Realising this objective requires that the appraisal mechanism should have many facets and be non-judgmental. An informal and continuous dialogue between trainer and trainee is vital and often occurs in the clinical setting. Formal appraisals are held every six months with the following aims:

- to establish short and long term objectives for the specialist registrar;

- to allow frank discussion about the specialist registrar's strengths and weaknesses;

- to agree a written record of the specialist registrar's progress, suitable for review by the programme director, the postgraduate dean, and the regional specialty education committee.

The appraisal is time limited to about one hour and is held in an undisturbed environment. Commitment on the part of both the trainer and trainee is a prerequisite for a worthwhile meeting. Both trainer and trainee prepare for the formal appraisal by completing documentation outlining the trainee's experience and achievements since the last meeting, together with information about the trainee's communication and teaching skills and any difficult or problem areas. Objectives for the next six months are decided.

Following the appraisal the trainer summarises the meeting and the resulting plans in a document signed and retained by both parties. A copy is also held by the programme director.

The opportunity for appraisal is also used in the half day training sessions when the supervising consultant considers trainees' participation in the session, their contribution to its planning, and their teaching ability and communication skills.

\section{Formal study time}

It is vital that the specialist registrar gains optimal benefit from the one or two half days allowed each week for study.

One half day each week, for three terms of 10 weeks every year, has been reserved for formal educational sessions. Sessions alternate between the two centres and are the responsibility of the educational supervisors.

Recognition of specialist registrars' educational needs, and the development of a training agreement between involved parties, is aided by the allocation of a specialist registrar to liaise with the educational supervisor in the planning and organisation of each session. Together they determine existing knowledge and skills among the trainees, thereby ensuring that the session is comprehensive enough to meet the needs of individuals. 
This approach allows trainees nearing the completion of training to demonstrate their teaching, leadership, and organisation skills, while enabling more junior trainees to develop such skills. When appropriate, the session may be taught by a specialist registrar with a particular area of expertise or interest.

Achieving a balance between the needs of trainees approaching the Fellowship examination and those embarking on their training is essential. This has been facilitated by the ease with which trainees have identified their educational needs. Senior trainees have focused upon management, administrative, and teaching skills, while their more junior colleagues have concentrated upon clinical matters, at the same time enjoying a flavour of future targets.

Attendance at these educational sessions is expected during the specialist registrar's secondments to other specialties.

During years 3,4 , and 5 of the programme a second half day is allocated for the specialist registrar to use for such activities as audit and research, writing papers and lectures, and for private study. A record of activities undertaken in this time is maintained.

\section{Structure of formal educational sessions}

The following structure accommodates the requirements of the Faculty of Accident and Emergency Medicine's guidelines for training in the specialty ${ }^{2}$ although it will be reviewed as the programme evolves.

The five year programme will include 90 clinical sessions, 30 management sessions, and 30 teaching/research/audit sessions. Sessions will follow a five week cycle:

- Week 1 Clinical-injury related topics

- Week 2 Management topics

- Week 3 Clinical-medicine and related topics

- Week 4 Teaching/research/audit topics

- Week 5 Clinical-other clinical specialties relevant to $\mathrm{A} \& \mathrm{E}$.

One of the teaching/research/audit sessions each term is devoted to specialist registrars presenting and evaluating their ongoing or proposed research and audit. The format of each session is designed to be flexible, with the following basic outline:

14.00-15.00 Teaching session by an "expert" 15.15-1600 Practical/skill based session, for example writing a business plan, $x$ ray interpretation, evaluation of clinical research 16.00-17.00 Interactive session, for example case discussions, OSCEs (objective structured clinical examinations)

\section{Implementation}

The specialist registrar training programme in accident and emergency medicine was introduced in Mid-Trent following meetings with the trainees. Those trainees who have remained in the senior registrar grade have chosen to participate in the specialist registrar training programme which has been developed.

All trainees have received documentation containing full details of the organisation of the programme, an outline record of the formal sessions and a copy of Guidelines for specialist training in accident and emergency medicine.

A personal outline of each trainee's rotations, secondments, and objectives is also to be included in the documentation.

\section{Assessment of training}

An annual assessment is conducted by the programme director and a representative of the regional postgraduate dean. This assessment considers the performance of both trainer and trainee together with the progress of the training programme, and facilitates completion of the record of in-training assessment (RITA) form. Unlike the appraisal mechanism, assessment is judgmental and may be both critical and congratulatory.

The following decisions result from the annual assessment:

- whether the trainee's objectives to date have been achieved;

- the suitability of the trainee to proceed to the next year of training;

- specific training needs of both trainee and trainer;

- further development and refinement of the training programme.

Feedback from the assessment to both trainee and trainer is constructive and tactful and acknowledges that not every consultant is a successful trainer. In Trent region there is currently a further review of all trainees by the Regional Specialty Education Committee.

\section{Discussion}

The success of the specialist registrar grade in accident and emergency medicine will be determined by many factors. Fundamental to its success is the commitment of trainees and their trainers to the establishment of training programmes which respond to the needs of the specialty. In turn, our training programme will be judged by its participants, future recruitment to the specialist registrar grade, and success in the Fellowship examination.

The programme we have developed will undoubtedly see many changes directed by the continuing challenges posed by the introduction of the specialist registrar grade. We hope in the short term it will stimulate new ideas among both specialist registrars and trainers.

\footnotetext{
1 Department of Health. A guide to specialist registrar training. London: Department of Health, March 1996.

2 Faculty of Accident and Emergency Medicine. Guidelines for specialist training in accident and emergency medicine. Prepared by the Board of the Faculty of Accident and Emergency Medicine, March 1996.
} 